\title{
Fractal structures in perovskite-based solar cells
}

\author{
B. L. Oksengendler ${ }^{1}$, N. R. Ashurov ${ }^{1}$, S. E. Maksimov ${ }^{1}$, I. Z. Uralov ${ }^{1}$, O. V. Karpova ${ }^{2,3}$ \\ ${ }^{1}$ Polymer Chemistry and Physics Research Center at The National University of Uzbekistan named after Mirzo \\ Ulugbek, 100128, A. Kadyri Str., 7b, Tashkent, Uzbekistan \\ ${ }^{2}$ Turin Polytechnic University in Tashkent, 17 Kichik Halka Yuli, Tashkent, 100095, Uzbekistan \\ ${ }^{3}$ Faculty of Physics, National University of Uzbekistan, Tashkent, Uzbekistan \\ oksengendlerbl@yandex.ru
}

PACS 05.45.Df

DOI 10.17586/2220-8054-2017-8-1-92-98

\begin{abstract}
Interface engineering plays important role in the fabrication of tandem and perovskite-based solar cells. Recent experiments show that the interface effects are caused by the coupling of the electron bands and the pairing of surface contact geometry. In particular, it has been experimentally revealed that the transition from a planar to a rough interface improves many photoelectric parameters of the device. This means that the value of the fractal dimension of the interface may be key factor in device performance. It is possible to formulate two problems: firstly, the understanding on simple models why the electrical properties are improved with fractal interfaces, and, secondly, to discuss one of the most promising approaches in modern electronics, namely technology of radiation applications in the creation of rough interfaces.
\end{abstract}

Keywords: solar cells, perovskite, interface, fractal.

Received: 27 July 2016

Revised: 2 September 2016

\section{Introduction}

Thin film photovoltaic materials based on polymers and tandem structures have attracted much attention due to their flexibility, environmental safety and low cost. However, to compete with silicon based solar cells, conversion efficiency and stability of organic and tandem solar cells need to be improved considerably. To address these concerns, in particular, perovskite based solar cells have been developed whose conversion efficiencies have monotonically increased over the last 3-4 years [1-3].

Fabrication of highly efficient solar cells (SC) based on organic-inorganic perovskites requires basic studies related to such issues as the physical and chemical aspects of cell morphology [4], the architecture of device structures [5], stability and degradation of photovoltaic materials and devices [6], thermodynamics and electronics of defects [7], homologous composition of perovskites [8], the role of the organic component of perovskites [9], mechanisms of photophysics [10] in organic and tandem structures.

All these issues can be classified as the "problem of interface engineering for photovoltaics" [11]. In this paper, we address the problem of rough interfaces in the charge dynamics and conversion efficiency of the perovskite based tandem solar cells. This problem is of special importance for perovskites because of their multi-component nature. Earlier, in the study of similar problem in thin film silicon solar cells, it was found that the surface "landscape" plays an important role causing the multiple reflection of the light [12]. Similar effects in organicinorganic perovskite solar cells were studied in [13], where the cells with rough interface surfaces were fabricated.

In particular, in [13], additional power conversion efficiency should be gained without increasing the thickness and the complexity of the devices for practical applications. This result has been checked in [13] for 20 samples. A rough interface between perovskite and hole-conducting material (HTM) was fabricated in perovskite solar cells to enhance the light scattering effect and improve the charge transport [13]. The parameters related to the morphology have been systematically investigated by sequential deposition. The control of the roughness degree of the interface was realized by two ways: a) reaction temperature control and b) pre-wetting time control (see, Fig. 1). Simultaneous enhancements of short-circuit current and power conversion efficiency were observed in both $\mathrm{CH}_{3} \mathrm{NH}_{3} \mathrm{PbI}_{3}$ and $\mathrm{CH}_{3} \mathrm{NH}_{3} \mathrm{PbI}_{3-x} \mathrm{Cl}_{x}$ devices containing the rough interface, with power conversion efficiencies of $10.2 \%$ and $10.8 \%$, respectively, additionally, the enhancement of the short-circuit current was more than $13 \%$.

Here it is important to note that the increase of the total surface for the fractal interface should decrease the current through the device, since the interface has electron traps. However, this is in contradiction with the experimental results of [13]. This may imply that the fractal structure at the interfaces plays an important role. Therefore, the theoretical analysis of such structures and their role in photovoltaic conversion is important for deeper understanding of the mechanisms for charge carrier generation and dynamics. 


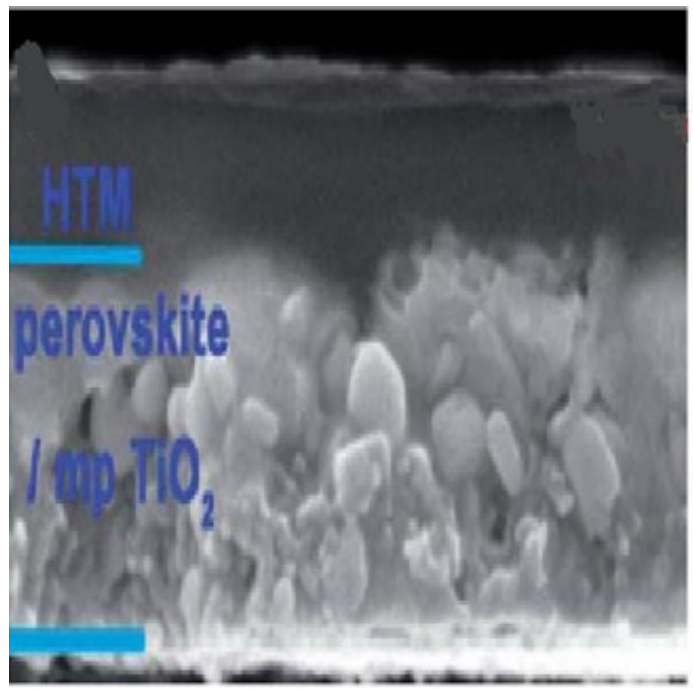

(a)

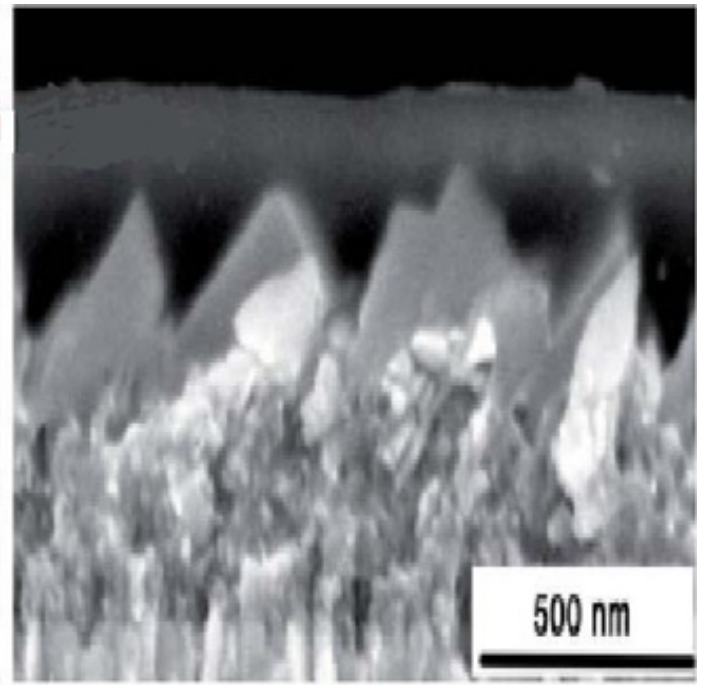

(b)

FIG. 1. Scanning electron microscope images of cross-sections of perovskite/HTM interface: (a) planar, (b) rough relief (a picture taken from [13])

These theoretical and experimental results highlight an efficient and universal way to control the morphology and further optimize perovskite solar cells for devices by sequential deposition with various structures.

\section{Light passage through the fractal interface}

The interaction of electromagnetic waves with the fractal ("rough") surfaces has attracted much attention earlier within different models. However, the geometric structures of the rough surfaces considered in these models are very far from that of the perovskite cells studied in [13]. Therefore, we consider a simple model of the "perovskite particles/ HTM film" interface, which is more close to the interface structures studied by the SEM method. Let us assume that the interface contains the tightly contacting perovskite balls with radii R and the dense HTM film. The light beam falls vertically on the interface inside perovskite cell from the side of the balls (see Fig. 2). It is obvious that there is a distance $\rho^{*}=Y_{0}$ measured from the central diameter of the ball (see, Fig. 2a), further, which the light beam undergoes the total internal reflection and remains inside the perovskite. From the simplest constructions, the following expression for the part of the total interactions of parallel rays with the spherical surface of the perovskite ball, when the light rays remain in the ball and cannot transit into the HTM film can be obtained: $F=\left(1-\eta^{2}\right)^{3 / 2}$ (here $\eta=\sin \alpha^{*}$ is the light refraction index in the transition from the more dense medium of perovskite into the less dense HTM medium).

Now, suppose that the interface is formed from the ellipsoidal perovskite particles (with semi-axes $a, b, c=b$ ) and the HTM film (Fig. 2b). The calculations for ellipsoidal particles are similar to those for the spherical particles and allow one to obtain the part of rays falling initially parallel to the major axis of the ellipsoid but undergoing total internal reflection: $\tilde{F}=\tilde{Z}^{3} / 2\left(1+\tilde{Z}^{2}\right)^{3 / 2}$; here $\tilde{Z}=\left(\frac{a}{b}\right) \operatorname{ctg} \alpha^{*}$. It is important to note that in the case of the spherical particles $(a=b)$, the expression for $\tilde{F}$ transforms into that of $F$. Therefore we can conclude that in the case of the surface consisting of ellipsoidal grains, the probability of total internal reflection of light beams is greater than in the case of spherical particles of perovskites. From this conclusion, it follows that the number of electron-hole pairs generated per one second in the subsurface particles of perovskite increases with increased interface roughness. Of course, this leads to the increase of the current through the interface. Note that these results are in agreement with the concept of "true fractal interfaces". Indeed, as a result of technological operations, a large set of perovskite particles with varying degrees of ellipticity (i.e. with different $\frac{a}{b}$ relations) is presented at the interface. If we introduce the idea about the value of the particle deformability, $\tilde{\beta}=2 \frac{a-b}{a+b}$, then at the small degrees of deformability, we obtain $\frac{a}{b} \approx 1+\tilde{\beta}$. In this way, in the expression for $\tilde{F}$ we can introduce the dependence on $\tilde{\beta}$, and then we consider it distributed in agreement with the fractal laws. This case corresponds 

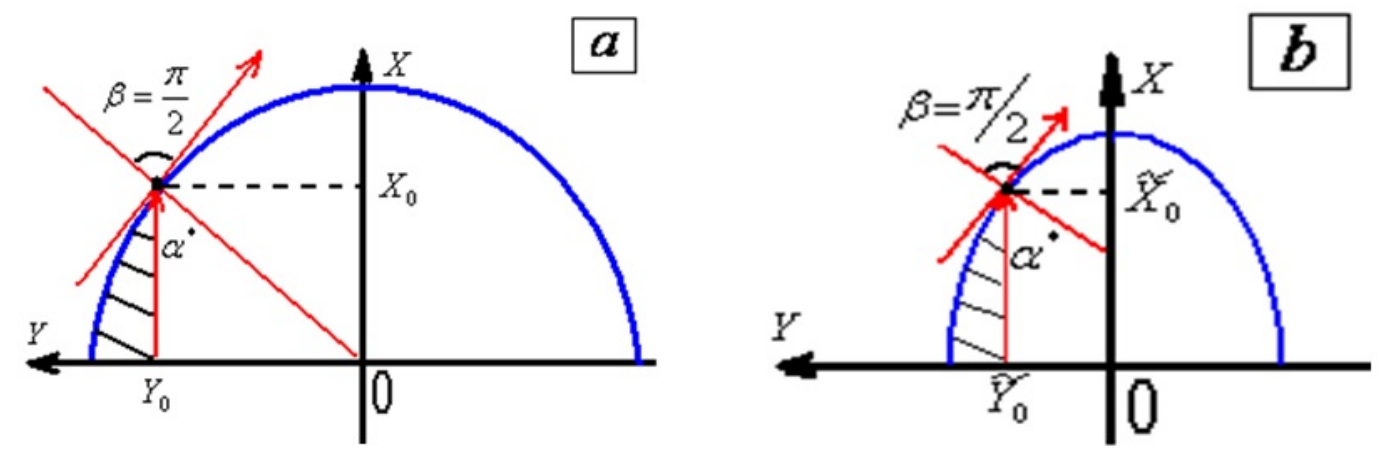

FIG. 2. Schematic representation for the total internal reflection inside the round (a) and ellipsoidal (b) particles of perovskite; in the case (b) the shaded area is greater than in (a) $\left(\frac{Y_{O}}{R}>\frac{\tilde{Y}_{O}}{R}\right)$, which corresponds to the larger number of generated $e$ - $h$-pairs for the ellipsoidal particles

to the change of the shape of perovskite particles under the influence of some type of technology, but with the preservation of its volume. Thus we can assume that the growth of the fractal dimension (roughness) of interface causes a decrease of the current due to the enhanced electron-hole recombination at the interface.

\section{Current on the fractal interface}

The effect of the interface fractal dimension on the photocurrent should be resolved in terms of the interaction of electron de Broglie waves with a fractal. Due to the lack of such investigations (see [14]), we restrict our study by considering the simple quantum mechanical model. Since the fractal interface is characterized by non-uniform density of matter, it is reasonable to use the approach "on average" for the first approximation when the interface can be characterized by a spatial value $2 a$, which is mean-square deviation of the value of roughness at the interface. Then $2 a \approx \sigma$ and the potential acting on electron can be chosen as $V(\tilde{x})=\frac{1}{2} V_{0}\left(1+\operatorname{th} \frac{\tilde{x}}{2}\right)[15,16]$ (see, Fig. 3). Then, for the transmission coefficient (of the wave through the interface), we have:

$$
\tilde{T}=1-\left[\frac{\operatorname{sh} \pi\left(k-k^{\prime}\right) a}{\operatorname{sh} \pi\left(k+k^{\prime}\right) a}\right]^{2} \approx 1-\left(\frac{\pi a V_{0}}{\hbar}\right)^{2} \frac{2 m}{E} \exp \left(-\frac{4 \pi a}{\hbar} \sqrt{2 m E}\right) .
$$

Here $V_{0}$ is the difference of the potential energy of electrons between the particles of perovskite and the film covering the interface: $k=\frac{2 m}{\hbar^{2}} E ; k^{\prime}=\frac{2 m}{\hbar^{2}}\left(E-V_{0}\right) ; E$ is the electron energy. The formula for $\tilde{T}$ demonstrates clearly that $d \tilde{T} / d a>0$. Since the interface fractal dimension $D$ is symbatical to $\sigma^{2}$, then $d \tilde{T} / d D=(d \tilde{T} / d a) \cdot(d a / d \sigma) \cdot(d \sigma / d D)>0$. This shows the electron transport through the interface is enhanced by increasing of its fractal dimension. It is important to note that the opposite difference of the potential relief for electrons facilitates the passage through the steps at its broadening in the direction perpendicular to the interface which is consistent with the experimental results of the Ref. [13].

\section{Controlling of the interface fractal structure by radiation}

Consider a planar boundary in the characteristic architecture of perovskite based solar elements (Fig. 4a).

The interface roughness (including the fractal structure) can be designed by ion bombardment. Earlier, such models have been discussed in the Refs. [17,18].

The interface between the surface of hole transporting material (HTM) and perovskites, made in the form of fine particles can be considered as quasi-plane (Fig. 4a). Such a "weakly rough" surface may be characterized by the surface height $(Z)$ at its different points along a certain direction. Then, a portion of representative surfaces can be characterized by the relation:

$$
\sigma^{2}=\left\langle Z^{2}(y)\right\rangle
$$

Here, the brackets denote averaging over the interface area, the reference point on a vertical is chosen such that $\langle Z(y)\rangle=0$. An important measure of the statistical properties for the surface is the correlation function given by:

$$
C(\Delta y)=\langle Z(y+\Delta y) * Z(y)\rangle \text {. }
$$




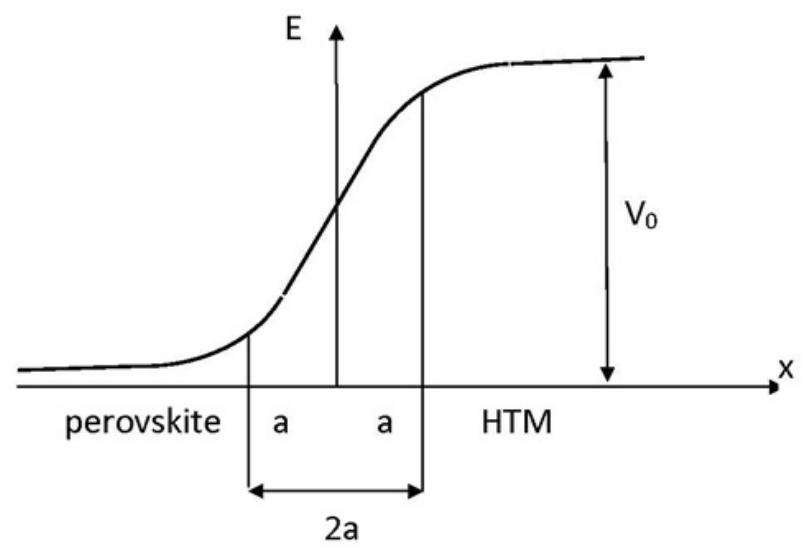

FIG. 3. Potential relief modelling the energy barrier at the transition of an electron through a "fuzzy" interface

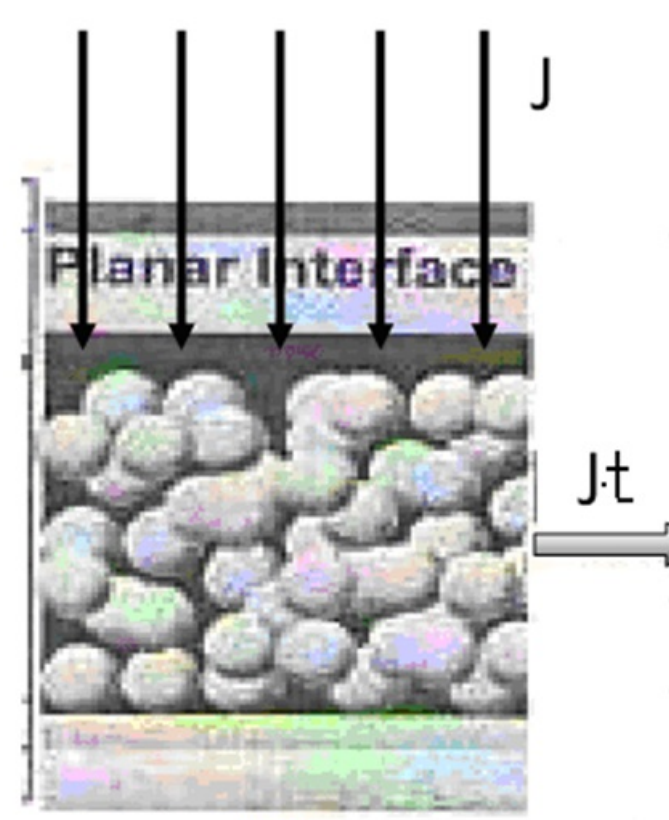

(a)

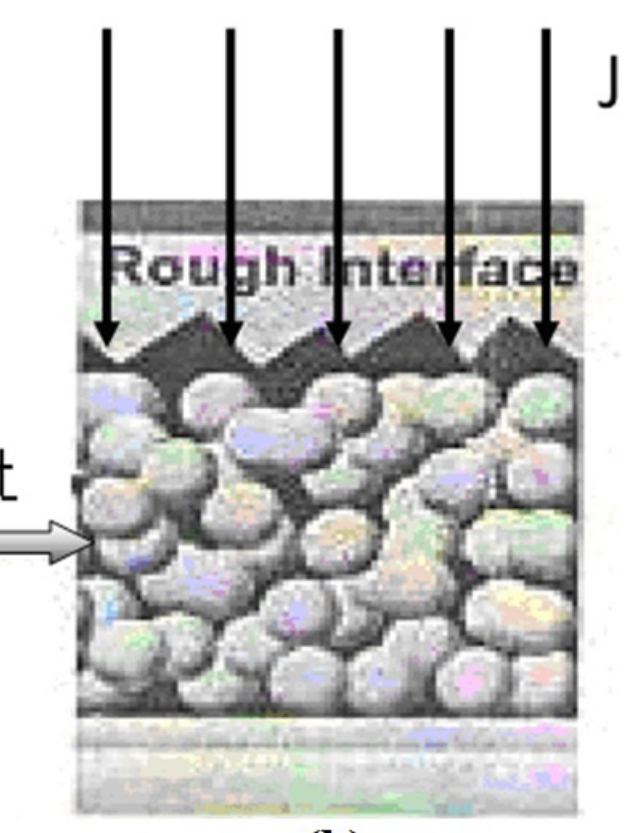

(b)

FIG. 4. Diagram of the perovskite solar cells on the planar (left) and rough (right) interface under irradiation with the intensity of ion irradiation $J$

It can be expressed in terms of "power spectrum" $G(f)$ by Fourier transformation:

$$
C(\Delta y)=\int_{f \min }^{\infty} G(f) \exp (2 \pi i f \Delta y) d f
$$

Here, $f$ is the spatial frequency which is inversely proportional to the wavelength corresponding to the rough surface. It is clear that $f_{\min }=1 / M_{\max } \cdot f_{\min }=1 / M_{\max }$, where $M_{\max }$ is the physical length of the entire interface. Choosing the "fractal spectrum" of power $\mathrm{G}(\mathrm{f})=\frac{\chi}{f^{\alpha}}$ (where $\chi$ is the coefficient surface irregularity), we have:

$$
\sigma^{2}=\left\langle Z^{2}(y)\right\rangle=\int_{f_{\min }}^{\infty} G(f) d f=\frac{\chi M_{\max }^{\alpha-1}}{(\alpha-1)}
$$


From the Mandelbrot relation [19] $\alpha=7-2 D$, we obtain the expression of fractal dimension $(D)$ for rough surface with its dispersion:

$$
D=3-\frac{\text { const }}{\sigma^{2}}
$$

Eq. (6) is valid in the interval of $2<D<32$ and was tested on a huge number of surface types (see, e.g., [19]).

Note that the constant in the last formula allows one to obtain the correct value of $D$ for extreme cases: perfectly smooth $(\sigma \rightarrow \infty)$ and absolutely rough $(\sigma \rightarrow \infty)$ surfaces; formally, it is sufficient in the upper limit of the integrals used to replace infinity $f_{\max }$, expressed in terms of the minimum size of a representative portion of the surface. Thus, to verify in the growth of $D$ under the action of radiation (Fig. 4b) it is necessary to prove an increase in the spread of height "hills" on the surface (i.e., increasing its roughness). Let us return to the original formulation of the problem and use the results of Mullins (1959) (see in [20]) to describe the evolution of the groove on the border of the two grains (Fig. 5).

Under the influence of the difference in the chemical potential $\Delta \mu=\gamma_{s} \Omega \tilde{K}$, for certain constraints, there is a material removal from the contact between two grains (in our case from two adjacent particles of perovskite) on the border of the groove (here, $\gamma_{s}$ is the surface tension of the free surface, $\Omega$ is the atomic volume, $\tilde{K}$ is the curvature of the surface). As a result, the groove depth $d$ increases over time.

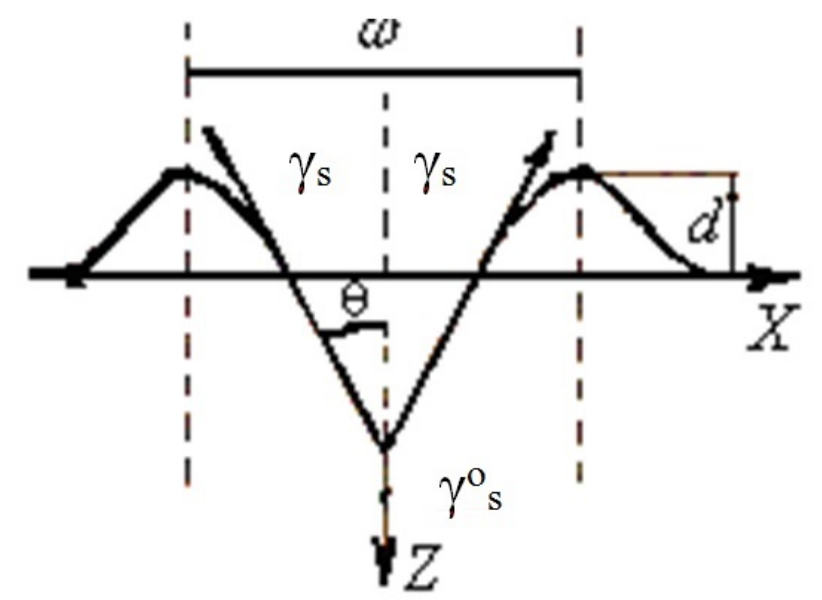

FIG. 5. Profile of "groove" in place of the output of the boundary of two particles on the surface of the interface under irradiation. (see [20])

For the case of surface diffusion, we obtain:

$$
d=2(\operatorname{ctg} \theta)(B t)^{1 / 4}
$$

where $B=\frac{D_{s} \delta \gamma_{s} \Omega}{k_{B} T}, D_{s}$ is the surface radiation enhanced atom diffusion coefficient, $\delta$ is the width of the surface layer having a high rate of diffusion; $\theta$ is the angle of the original groove before its recess (at $t=0$ ), (Fig. 5).

Thus, the value $d$ at different points of the surface is determined by the angle $\theta$ at a predetermined growth time $(t)$. When $\theta$ is increased from 0 to $\pi / 2$ rate of groove deepening decreases. The speed of growth is determined by depending on the irradiation parameters and increases with increasing $\delta$ and $D_{s}$ : managing them, you can change the value of $d$. Obviously, the larger $B$, the difference in the initial increases $\{d\}$, i.e. it increases the degree of roughness and hence the fractal dimension $D$. This qualitative picture can easily be translated into the language of mathematics, if we take into account that the original set of $\theta$ has a different fractal distribution, averaging on which allows to give all the necessary statistical values.

\section{Photodegradation of solar cells with fractal interfaces}

Since the presence of fractal interface leads to its excessive area, the exposure by ionizing light (as such as by any type of ionizing radiation in general), taking into account the semiconductor nature of the perovskite, can lead to the surface radiation-stimulated atomic diffusion. This diffusion takes place by recharge of local electron levels and should lead to the smoothing of the roughness of the interface; this, in turn, should eliminate the extra value 
of $13 \%$ of the photocurrent. The effect of radiation smoothing of fractal has been observed already on a $\mathrm{BaF}_{2}$ surface [21].

However, to realize such surface smoothing by surface atom diffusion, it is necessary to release them from their regular surface layer of perovskite, i.e. the action of some mechanism for the formation of defects on the surface under exposition of light, UV-radiation (as well as the X- and gamma-rays in the case of work of device in space) must take place. The most important mechanism of the radiation defect may be the so-called mechanism of Dexter-Varley, including his variations in form of Knotek-Feibelman [22]. Its essence is the ionization of deep shell of surface negative ion by X-rays, resulting that the deep-level hole rises to higher levels by Auger transition with "breeding"; the negative ion is converted into the positive one losing electrons (in our case it is $I_{s}^{-} \rightarrow I_{s}^{+}$). Such a transformation makes the ion $I_{s}^{+}$Coulomb unstable and it then leaves its site. This process is probabilistic in nature and is governed by the ratio of the reverse Debye frequency $1 / \omega_{D}$ and of the time $\tau_{e}$ of life of hole on the $I_{s}^{+}$ion, so that the cross section of the destruction is proportional to $\eta \sim \exp \left(-\frac{1}{\omega_{D} \tau_{e}}\right)$ (see [22]). Since the surface ions $I_{s}^{-}$form area of Tamm states [23], the delocalization of the holes takes place on Tamm zone, so $\tau_{e} \approx \frac{\hbar}{\Delta E_{T}}$, where $\Delta \mathrm{E}_{T}$ is the width of the Tamm zone [23]. Turning to the fractal surface of the interface where the convexes alternate with the cavities (see. Fig. 1), we see that electron wave functions overlap on neighboring ions $I_{s}^{-}$in the areas of convexity worse than on a flat surface, whereas in the area of the cavities the situation is opposite. The result is the variation of $\Delta E_{T}$ on the convexities and the concavities, but the time $\tau_{e}$ is higher on the convexities than on the concavities. This, in turn, leads one to the conclusion that the probability of convex domain destruction is greater. Consequently, under the X-rays (and under the corresponding UV-radiation) the smoothing of the relief takes place that reduces the fractal dimension of the interface and leads to the degradation of the device as lost an additional $13 \%$ found in [13].

\section{Conclusion}

In this paper, we proposed a model which explains experimentally established effect of photovoltaic parameter enhancement for perovskite-based solar cells in the transition from a planar to a rough interface. The model is based on the assumption that the surface perovskite interface has the fractal structure that may cause an increase in the mobility of the electrons. The rigorous analysis the problem can be done by modeling the interaction of the waves of different nature with fractal surfaces. The above model can be used for the comprehensive description of the problem of charge carrier generation, separation and transport in perovskite-based tandem solar cells.

\section{Acknowledgements}

This work was supported by the Committee for Coordinating the Development of Science and Technology under the Cabinet of Ministers of the Republic of Uzbekistan, contract No. F3-003.

\section{References}

[1] Giorgi G., Yamashita K. Organic-inrganic perovskites: an ambipolar class of materials with enhanced photovoltaic performances. J. Mater. Chem. A, 2015. DOI: 10.1039/c4ta05046k.

[2] Yin W.-J., Yang J.H., Kang J. et al. Halide perovskite materials for solar cells: a theoretical review. J. Mater. Chem. A, 2014. DOI: V. 10. 1039/c4ta05033a.

[3] Ashurov N.R., Oksengendler B.L., Rashidova S.Sh., Zakhidov A.A. State and Prospects of Solar Cells Based on Perovskites. Appl. Solar Energy, 2016, 52 (1), P. 5-15.

[4] Salim T., Sun S., Abe J., et.al. Perovskite-based solar cells: impact of morphology and device architecture on device performance. J. Mater. Chem. A, 2015. DOI: 10.1039/c4ta05226a.

[5] Liu Ch., Oiu Zh., Meng W. et.al. Effect of interfacial characteristics on photovoltaic performance in $\mathrm{CH}_{3} \mathrm{NH}_{3} \mathrm{PbBr}_{3}-$ based bulk perovskite solar cells with core/shell nanoarray as electron transporter. Nano Energy, 2015, 12, P. 59-68.

[6] Oksengendler B.L., Ismailova O.B., Marasulov M.B, Urolov I.Z. On the degradation mechanism of functioning solar cells based on organic-inorganic perovskites. Appl. Solar Energy, 2014, 50 (4), P. 255-259.

[7] Du M.H. Efficient carrier transport in halide perovskites: theoretical perspectives. J. Mater. Chem. A, 2014, 2, P. 9091-9098.

[8] Shkrob I., Marin T. Charge trapping in photovoltaically active perovskites and related halogenoplumbate compounds. J. Phys. Chem. Lett., 2014, 5, P. 1066-1071.

[9] Frost J., Butler K., Brivo F., et. al. Atomistic origins of high-performance in hybrid halide perovskite solar cells. arXiv: 1042. 4980v3[Cond.-Mat.mtrl-sci] apl, 2014.

[10] Sum T., Mathews N. Advancements in perovskite solar cells: photophysics behind the photovoltaics. Energy and Environ. Sci., 2014, 7, P. 2518-2534.

[11] Graetzel M., Janssen R., Mitzi D., Sargent E.H. Materials interface engineering for solution-processed photovoltaics. Nature, 2012, 488, P. 304-312.

[12] Mueller J., Rech B., Springer J., Vanecek M. TCO and light trapping in silicon thin film solar cells. Solar Energy, 2004, 70, P. 917-930. 
[13] Zheng L., Ma Y., Chu S., et.al. Improved light absorbtion and charge transport for perovskite solar cells with rough interfaces by sequential deposition. Nanoscale, 2014, 6, P. 8171-8176.

[14] Jakeman E., in: Pietronero L., and Tosatti E., (ed.) Fractals in Physics. North Holland, Amsterdam-Oxford-New York-Tokyo, 1986, p. 82.

[15] Flugge S. Practical Quantum Mechanics I, Springer-Verlag, Berlin-Heidelberg-New-York, 1971,99 p.

[16] Bohm D. Quantum Theory. Prentice-Hall Ing, New-York, 1952, 722 p.

[17] Parilis E., Kishinevskiy L., Turaev N., et al. Atomic Collisions on Solid Surfaces. Elsevier Sci. Publ. North. Holl., Amsterdam, London, New York, Tokyo, 1993, 664 p.

[18] Maksimov S.E., Oksengendler B.L. Turaev N.Yu. Synergetic Approach to Studying Material Sputtering under Ion Bombardment. Journ. of Surf. Invest., 2013, 7 (2), P. 333-338.

[19] Feder E. Fractals. Plenum Press, New York, 1988, 262 p.

[20] Shewmon P.G. Diffusion in solids. McGraw-Hill Book Com., NY, San Francisco, Toronto, London, 1961,189 p.

[21] Yadav R.P., Kumar M., Mital A., et al. Fractal and multifractal characteristics of swift heavy ion induced self-affine nanostructured BaF 2 thin film surfaces. Chaos, 2015, 25, P. 083115.

[22] Oksengendler B.L., Maksimov S.E., Marasulov M.B. Degradation of perovskites and Dexter-Varley paradox. Nanosystems: physics, chemistry, mathematics, 2015, 6 (6), P. 825-832.

[23] Tamm I.E. Collection of scientific works in two volumes. Moscow: Nauka, 1975, V.1. (in Russian) 